\title{
The neurocognitive function change criteria after whole-brain radiation therapy for brain metastasis, in reference to health- related quality of life changes: a prospective observation study
}

Toshimichi Nakano 1* (D), Hidefumi Aoyama ${ }^{1 *}$, Hirotake Saito², Satoshi Tanabe², Kensuke Tanaka?, Katsuya Maruyama', Tomoya Oshikane ${ }^{1}$, Atsushi Ohta², Eisuke Abe ${ }^{1}$ and Motoki Kaidu ${ }^{1}$

\begin{abstract}
Background: We sought to construct the optimal neurocognitive function (NCF) change criteria sensitive to healthrelated quality of life (HR-QOL) in patients who have undergone whole-brain radiation therapy (WBRT) for brain metastasis.

Methods: We categorized the patients by the changes of NCF into groups of improvement versus deterioration if at least one domain showed changes that exceeded the cut-off while other domains remained stable. The remaining patients were categorized as stable, and the patients who showed both significant improvement and deterioration were categorized as 'both.' We examined the clinical meaning of NCF changes using the cut-off values 1.0, 1.5, and 2.0 SD based on the percentage of patients whose HR-QOL changes were $\geq 10$ points.

Results: Baseline, 4-month and 8-month data were available in 78, 41 (compliance; 85\%), and 29 (81\%) patients, respectively. At 4 months, improvement/stable/deterioration/both was seen in 15\%/12\%/41\%/32\% of the patients when 1.0 SD was used; $19 \% / 22 \% / 37 \% / 22 \%$ with 1.5 SD, and $17 \% / 37 \% / 37 \% / 9 \%$ with 2.0 SD. The HR-QOL scores on the QLQ-C30 functional scale were significantly worse in the deterioration group versus the others with $1.0 \mathrm{SD}$ ( $p=$ $0.013)$ and $1.5 \mathrm{SD}(p=0.015)$. With $1.5 \mathrm{SD}$, the HR-QOL scores on the QLQ-BN20 was significantly better in the improvement group versus the others $(p=0.033)$. However, when 'both' was included in 'improvement' or 'deterioration,' no significant difference in HR-QOL was detected.
\end{abstract}

Conclusions: The NCF cut-off of 1.5 SD and the exclusion of 'both' patients from the 'deterioration' and 'improvement' groups best reflects HR-QOL changes.

Keywords: Brain metastasis, Health-related quality of life, Neurocognitive function, Whole brain radiation therapy

\footnotetext{
*Correspondence: t-nakano@med.niigata-u.ac.jp; h-aoyama@med.niigata-

u.ac.jp

${ }^{1}$ Department of Radiology and Radiation Oncology, Niigata University

Graduate School of Medical and Dental Sciences, 1-757 Asahimachi-dori,

Chuo-ku, Niigata 951-8510, Japan

Full list of author information is available at the end of the article
}

(c) The Author(s). 2020 Open Access This article is distributed under the terms of the Creative Commons Attribution 4.0 International License (http://creativecommons.org/licenses/by/4.0/), which permits unrestricted use, distribution, and reproduction in any medium, provided you give appropriate credit to the original author(s) and the source, provide a link to the Creative Commons license, and indicate if changes were made. The Creative Commons Public Domain Dedication waiver (http://creativecommons.org/publicdomain/zero/1.0/) applies to the data made available in this article, unless otherwise stated. 


\section{Background}

Brain metastases are a common sequela of cancer, occurring in approx. 24-45\% of all cancer patients [1]. Neurocognitive function (NCF) is considered to reflect the status of the brain tumor burden as well as the degree of the adverse radiation effect on the brain $[2,3]$. The combination of the Hopkins Verbal Learning Test-Revised (HVLT-R), the Trail-Making Test (TMT), and the Control Oral Word Association Test (COWAT) is a standardized neurocognitive battery, proposed by the Response Assessment in NeuroOncology (RANO) Group [4].

However, NCF change score criteria are not uniform across studies [5], and the proportion of patients reported to be suffering from neurocognitive deterioration after whole-brain radiation therapy (WBRT) has thus varied widely among studies, from 52 to $91.7 \%$ [6, 7]. It is also difficult to interpret whether the deterioration at 3-4 months after radiation is clinically meaningful, because of numerous confounding factors. We examined the validity of $\mathrm{SD}(1.0,1.5$, or 2.0$)[6,8]$, which are employed in the previous major trials.

We designed an observational study to address these issues, using successively collected NCF and health-related quality of life (HR-QOL) data in patients who underwent WBRT for brain metastases. Our study objectives were to clarify the appropriate NCF change criteria reflecting clinically meaningful changes in HR-QOL.

This is the first-ever study using the Japanese version of the RANO-proposed battery for the assessment of NCF and HR-QOL.

\section{Methods}

\section{Study population and treatment}

Patients were eligible if they were $\geq 18$ years old and had one or more brain metastases and were scheduled to undergo WBRT. The recruitment of patients took place between April 2012 and March 2017. The exclusion criteria were: Karnofsky Performance Status (KPS) <60, and severe neurological deficits that could interfere with the administration of the NCF and HR-QOL examinations. Written informed consent was obtained from all patients. This study was approved by the Institutional Review Board (Study \#1449).

\section{The assessment of neurocognitive function and health- related quality of life}

The patients' NCF was assessed using the Japanese versions of the HVLT-R, TMT, and COWAT. The HVLT-R consists of three domains, i.e., Total Recall (TR), Delayed Recall (DR), and Delayed Recognition (DRec), which are related to immediate and learning memory, delayed memory, and recognition, respectively. Parts A and B of the TMT (TMTA) and (TMT-B) assess an individual's processing speed and executive function, respectively. The COWAT assesses semantic fluency.

We converted the patients' raw individual test scores into standardized $\mathrm{z}$-scores by using the means and standard deviations (SDs) of individually age- and gender-matched healthy controls $[9,10]$. We selected changes in the z-score of $\geq 1.0,1.5$, and 2.0 of SD to examine how the differences in the cut-off value may affect the HR-QOL values $[6,8,11]$.

The patients' HR-QOL was assessed with two meansures: the European Organization for Research and Treatment of Cancer Quality of Life Questionnaire Core 30 (EORTC QLQ-C30) ver. 3.0 [12] and the Brain Cancer Module (QLQ-BN20) [13]. The QLQ-C30 is composed of one scale measuring an individual's global health status (GHS), five functional scales (physical, role, social, emotional, and cognitive functioning), and nine symptom scales (fatigue, nausea/vomiting, pain, dyspnea, insomnia, appetite loss, constipation, diarrhea, and financial difficulties). The QLQ-BN20 is a disease-specific module for brain cancer patients, and it consists of 11 symptom scales: future uncertainty, visual disorder, motor dysfunction, communication deficit, headaches, seizures, drowsiness, itchy skin, hair loss, weakness of legs, and bladder control. All raw scores were linearly transformed and scored from 0 to 100 according to the guidelines. A higher score on the GHS scale or the functioning scales indicates higher quality of life, whereas a higher score on the symptom scales indicates poorer quality of life.

We used the patients' NCF test scores and HR-QOL questionnaire responses obtained at baseline, 4 months, and 8 months after WBRT in this study.

\section{Statistical analyses \\ At baseline}

The patients' NCF and HR-QOL scores are presented as mean scores. We compared the scores between groups based on age (<65 years old vs. $\geq 65$ years old), KPS (100-80 vs. 70-60), the patients' number of brain metastases (1-4 vs. $\geq 5$ or meningeal carcinomatosis, the Graded Prognostic Assessment (GPA) (4.0-2.0 vs. 1.51.0) [14], the number of examinations (1: baseline-only data were available vs. $\geq 2$ : the baseline plus 4-month data, or the baseline, 4-month, and 8-month data were available), the use of surgical resection for brain metastases, and the systemic therapies prior to WBRT. We used the Mann-Whitney test for the individual NCF domains and the HR-QOL scales.

\section{At 4 months and 8 months}

NCF and HR-QOL Regarding the domain level, we assigned the changes in the score on each NCF domain over the time periods of the baseline to 4 months and 
the baseline to 8 months to one of three categories: 'improvement,' 'stable,' or 'deterioration.' Improvement and deterioration were defined as an increase or decrease in the score over the cut-off value (1.0, 1.5, or $2.0 \mathrm{SD})$. Other changes were defined as 'stable.'

Regarding the patient level, we classified the patients into four categories: 'improvement,' 'deterioration,' 'stable,' and 'both.' 'Improvement' and 'deterioration' were defined as exhibiting significant improvement or deterioration in NCF on at least one domain while other domains remained stable. The category of 'stable' was defined as no significant changes in any domain, whereas the 'both' category included the patients who showed both significant improvement and deterioration, in different domains.

Regarding the patients' HR-QOL, the $\geq 10$-point changes of each scale at 4 months and 8 months were assigned to one of three categories: 'improvement,' 'stable,' or 'deterioration' for individual HR-QOL scales. The same categorization was done for the mean QLQ-C30 functional/symptoms scales and the QLQBN20 scales.

The relationship of the changes in NCF and HR-QOL at 4 months We compared the percentages of deterioration and improvement on HR-QOL scales at 4 months postWBRT between groups at the patient level (deterioration vs. others, deterioration + both vs. others, improvement vs. others, and improvement + both vs. others) using the three cut-off values of 1.0, 1.5, and 2.0 SD. We used Fisher's exact test to examine the differences in the independent HR-QOL scales and the differences in the means QLQ-C30 functional/symptom scales and QLQBN20 scales.

The factors related to the deteriorations in NCF and HR-QOL at 4 months To examine the deterioration of NCF and that of HR-QOL from baseline to 4 months, we compared the percentage of deterioration on the NCF domains, GHS scale, and the means QLQ-C30 functional/symptom scales and QLQ-BN20 scales between groups based on age ( $<65$ vs. $\geq 65$ years old), GPA (4.0-2.0 vs. $1.5-0.0)$, the number of examinations (two examinations [baseline and 4 months] vs. three examinations [baseline, 4 months, and 8 months]), intracranial control at 4 months (progressive disease [PD] vs. non-PD), the systemic therapies prior to WBRT, the cytotoxic chemotherapy after WBRT, and the molecular targeted therapy after WBRT, using Fisher's exact test.

Overall survival was assessed by the Kaplan Meier-method. A $p$-value $<0.05$ was considered significant. All statistical analyses were performed with SPSS ver. 25 software.

\section{Results}

\section{The patients' characteristics}

The characteristics of the 78 consecutive patients at baseline and the 41 patients who underwent the examinations at 4 months are listed in Tables 1 and 2. The examinations compliance at 4 months and 8 months were high at $85 \%(41 / 48)$ and $81 \%(29 / 36)$, respectively. The median survival time (MST) of all patients was 7.3 months (95\% confidence interval: 4.2-10.4), 2.6 months (1.8-3.4) in the baseline-only group, $8.2(6.1-10.3)$ in the baseline plus 4-month examinations group, and 26.6 months (21.8-31.4) in the baseline, 4-month and 8month examination group (Additional file 1: Figure S1).

\section{The baseline NCF and HR-QOL data}

The mean z-score was -1.46 for the TR, -1.75 for the DR, -1.07 for the DRec, -0.46 for the COWAT, -1.46 for the TMT-A, and -1.12 for the TMT-B. The mean score for each HR-QOL scale are provided in Additional file 2: Table $\mathrm{S} 1$. Among the factors examined, only poor KPS of 60-70 (vs. 80-100) was associated with significantly poor NCF in two domains. Regarding the HR-QOL (total 26 scales), significantly poor scores were observed in the patients with a KPS of $60-70$ on 16 scales, or a GPA of $0-1.5$ on 11 scales; age $\geq 65$ years on five scales, the availability of examination (baseline-only) in three scales, undergoing surgery in three scales, the systemic therapies prior to WBRT in three scales; and the number of metastases $\geq 5$ or meningeal carcinomatosis on two scales. (Additional file 2: Table S1).

\section{The NCF and HR-QOL data at 4 months and 8 months The changes in the patients' NCF and HR-QOL}

At the domain level, regarding the changes in the NCF of the patients at 4 months after they underwent WBRT, when we used the cut-off value of 1.0 SD, NCF improvement was documented in $12-20 \%$ (TR/12\%, DR/20\%, DRec/12\%, COWAT/17\%, TMT-A/17\%, TMT-B/17\%) of the patients, stable NCF was observed in 43-69\% (TR/54\%, DR/43\%, DRec/47\%, COWAT/69\%, TMT-A/ $51 \%$, TMT-B/59\%), and deterioration was exhibited by 14-41\% (TR/34\%, DR/37\%, DRec/41\%, COWAT/14\%, TMT-A/32\%, TMT-B/24\%) compared to the baseline. The data at 8 months were $10-24 \%$ improvement (TR/ $14 \%$, DR/21\%, DRec/24\%, COWAT/21\%, TMT-A/10\%, TMT-B/17\%), 52-69\% stable (TR/62\%, DR/55\%, DRec/ 48\%, COWAT/65\%, TMT-A/69\%, TMT-B/52\%), and $14-31 \%$ deterioration (TR/24\%, DR/24\%, DRec/28\%, COWAT/14\%, TMT-A/21\%, TMT-B/31\%). With the cut-off value $1.5 \mathrm{SD}, \mathrm{NCF}$ improvement was documented in $6-17 \%$ of the patients, stable NCF was observed in $56-80 \%$, and deterioration was observed in $14-29 \%$ at 4 months, and the corresponding values at 8 months were $10-24 \%, 55-79 \%$, and $7-24 \%$, respectively. With the cut-off values of $2.0 \mathrm{SD}$, these values were $3-$ 
Table 1 The characteristics of the 78 patients who underwent whole-brain radiation therapy

\begin{tabular}{|c|c|}
\hline \multicolumn{2}{|l|}{ Age, yrs.; mean (SD): } \\
\hline Total & $61.8(11.5)$ \\
\hline$<65$ & $45(58)$ \\
\hline$\geq 65$ & $33(42)$ \\
\hline \multicolumn{2}{|l|}{ Gender: } \\
\hline Male & $43(55)$ \\
\hline Female & $35(45)$ \\
\hline \multicolumn{2}{|l|}{ Primary cancer: } \\
\hline Lung, non-small-cell & $36(46)$ \\
\hline Breast & $12(15)$ \\
\hline Lung, small-cell & $6(8)$ \\
\hline Others & $24(31)$ \\
\hline \multicolumn{2}{|l|}{ KPS: } \\
\hline $100-90$ & $26(33)$ \\
\hline 80 & $24(31)$ \\
\hline 70 & $16(21)$ \\
\hline 60 & $12(15)$ \\
\hline \multicolumn{2}{|l|}{ No. of brain metastases: } \\
\hline Solitary & $17(22)$ \\
\hline Oligo (2-4) & $16(21)$ \\
\hline Multiple $(5=<)$ & $34(43)$ \\
\hline Meningeal carcinomatosis & $11(14)$ \\
\hline \multicolumn{2}{|l|}{ GPA: } \\
\hline $4.0-3.0$ & $5(6)$ \\
\hline $2.5-2.0$ & $20(26)$ \\
\hline $1.5-1.0$ & $32(51)$ \\
\hline $0.5-0.0$ & $21(27)$ \\
\hline \multicolumn{2}{|l|}{ WBRT schedule: } \\
\hline $35 \mathrm{~Gy} / 14 \mathrm{fr}$ & $63(81)$ \\
\hline $30 \mathrm{~Gy} / 10 \mathrm{fr}$ & $7(9)$ \\
\hline Others & $5(6)$ \\
\hline Uncompleted & $3(4)$ \\
\hline \multicolumn{2}{|l|}{ Treatment: } \\
\hline WBRT only & $47(60)$ \\
\hline WBRT + SRS & $11(14)$ \\
\hline WBRT + Surgery (+/-SRS) & $20(26)$ \\
\hline \multicolumn{2}{|l|}{ Systemic therapies prior to WBRT } \\
\hline No & $23(29)$ \\
\hline Yes & $55(71)$ \\
\hline Cytotoxic chemotherapy & $50(64)$ \\
\hline Molecular targeted therapy & $25(32)$ \\
\hline Hormonal therapy & $7(9)$ \\
\hline Immunotherapy & $2(3)$ \\
\hline
\end{tabular}

No. of examinations:
Table 1 The characteristics of the 78 patients who underwent whole-brain radiation therapy (Continued)

\begin{tabular}{ll}
\hline Age, yrs.; mean (SD): & \\
\hline Total & $61.8(11.5)$ \\
\hline 1: BL only & $37(48)$ \\
2: BL and 4 mos. & $12(15)$ \\
3: BL, 4 mos. and 8 mos. & $29(37)$ \\
Examination compliance:\%, ( ): & \\
At 4 mos. & $85 \%(41 / 48)$ \\
At 8 mos. & $81 \%(29 / 36)$
\end{tabular}

Data are $n$ (\%) unless otherwise noted. KPS Karnofsky Performance Status, GPA Graded Prognosis Assessment, WBRT whole-brain radiotherapy, SRS stereotactic radiosurgery, $B L$ baseline, $P D$ progressive disease

$15 \%, 66-86 \%, 11-24 \%$ at 4 months, and $3-17 \%, 66-94 \%$, and $3-17 \%$ at 8 months, respectively (Fig. 1).

At the patient level, the percentage of patients who exhibited deterioration, improvement, both, or were stable with the use of different cut-off values (1.0 SD, 1.5 $\mathrm{SD}$, and 2.0 SD) are summarized in Fig. 1. At 4 months, improvement/stable/deterioration/both was seen in 15\%/ $12 \% / 41 \% / 32 \%$ of the patients when $1.0 \mathrm{SD}$ was used as the cut-off, $19 \% / 22 \% / 37 \% / 22 \%$ of the patients when 1.5 SD was used, and $17 \% / 37 \% / 37 \% / 9 \%$ of the patients when 2.0 SD was used. It is of note that the 'both' percentage was reduced from $32 \%$ with the cut-off value of $1.0 \mathrm{SD}$ to $22 \%$ with $1.5 \mathrm{SD}$, and further reduced to $9 \%$ with 2.0 SD. At 8 months, improvement/stable/deterioration/both was seen in $31 \% / 4 \% / 34 \% / 31 \%$ of the patients when 1.0 SD was used as the cut-off, 35\%/10\%/45\%/10\% with 1.5 SD, and $28 \% / 38 \% / 31 \% / 3 \%$ with $2.0 \mathrm{SD}$.

Regarding the HR-QOL at the scale level, at 4 months (and 8 months), improvement/stable/deterioration was seen $34 \% / 37 \% / 29 \%$ (and $38 \% / 45 \% / 17 \%$ ) in the GHS scale, $37 \% / 34 \% / 29 \%$ (and $41 \% / 35 \% / 24 \%$ ) in the mean QLQ-C30 functional scales, 27\%/32\%/41\% (and 28\%/ $51 \% / 21 \%)$ in the mean QLQ-C30 symptom scales, and $20 \% / 43 \% / 37 \%$ (and 10\%/59\%/31\%) in the mean QLQBN20 scales (Fig. 2).

\section{The relationship between the changes in NCF and HR-QOL at 4 months}

The scales on which the 'NCF-deterioration group' was significantly worse than the other statuses were physical function with $2.0 \mathrm{SD}$ as the cut-off, and social function, nausea and vomiting, and visual disorder with 1.0 SD. The scales on which the 'NCF-improvement group' was significantly better than the other statuses were social function and future uncertainty with 1.5 SD (Additional file 2: Table S2). Regarding the mean QLQ-C30/BN-20 scales, the mean QLQ-C30 functional scale score was significantly worse in the NCF-deterioration group than the other statuses with 1.0 SD $(p=0.013)$ and 1.5 SD 
Table 2 The characteristics of the 41 patients who underwent the examinations at 4 months

\begin{tabular}{ll}
\hline Age, yrs.; mean (SD): & \\
\hline Total & $63.0(11.4)$ \\
\hline$<65$ & $21(51)$ \\
$\geq 65$ & $20(49)$ \\
Gender: & \\
Male & $19(46)$ \\
Female & $22(54)$
\end{tabular}

Primary cancer:

Lung, non-small-cell

Breast

$24(59)$

Lung, small-cell

Others

KPS:

100-90

80

70

60

No. of brain metastases:

Solitary

Oligo (2-4)

Multiple $(5=<)$

Meningeal carcinomatosis

GPA:

$4.0-3.0$

$2.5-2.0$

$1.5-1.0$

$0.5-0.0$

WBRT schedule:

$35 \mathrm{~Gy} / 14 \mathrm{fr}$

$30 \mathrm{~Gy} / 10 \mathrm{fr}$

Others

Treatment:

WBRT only

WBRT + SRS

WBRT + Surgery (+/-SRS)

Systemic therapies prior WBRT

No

Yes

Cytotoxic chemotherapy

Molecular targeted therapy

Hormonal therapy

Immunotherapy

Systemic therapies after WBRT
Table 2 The characteristics of the 41 patients who underwent the examinations at 4 months (Continued)

\begin{tabular}{ll}
\hline Age, yrs.; mean (SD): & \\
\hline Total & $63.0(11.4)$ \\
\hline Yes & $35(85)$ \\
Cytotoxic chemotherapy & $19(46)$ \\
Molecular targeted therapy & $19(46)$ \\
Hormonal therapy & $2(5)$ \\
Immunotherapy & $3(7)$ \\
Intracranial control at 4 mos. & \\
PD & $13(32)$ \\
Non-PD & $26(63)$ \\
Unknown & $2(5)$ \\
\hline
\end{tabular}

Data are $n$ (\%) unless otherwise noted. KPS Karnofsky Performance Status, GPA Graded Prognosis Assessment, WBRT whole-brain radiotherapy, SRS

stereotactic radiosurgery, $B L$ baseline, $P D$ progressive disease
$15(37)$

14 (34)

$7(17)$

$5(12)$

$10(24)$

$12(29)$

$14(34)$

$5(12)$

$4(10)$

$13(32)$

18 (44)

$6(14)$

$36(88)$

1 (2)

$4(10)$

$20(49)$

$7(17)$

$14(34)$

16 (39)

$25(61)$

$22(54)$

$11(27)$

4 (10)

$1(2)$

$6(15)$
( $p=0.015$ ), but not significantly different with $2.0 \mathrm{SD}$. The mean QLQ-BN20 scale score was significantly better in the NCF-improvement group than the others only with 1.5 SD $(p=0.033)$. However, when 'both' was included in either 'deterioration' or 'improvement,' no significant difference in HR-QOL was detected (Table 3).

The factors related to the deterioration of NCF and HR-QOL at 4 months

When the cut-off value of $1.5 \mathrm{SD}$ was used, the significant factors for the deterioration of the patients' $\mathrm{NCF}$ test at 4 months were (1) intracranial tumor control (non-PD vs. PD) in two domains, and (2) the availability of 8-month examination data in four domains. The significant factor for the deterioration of the patients' HR-QOL at 4 months were the availability of 8-month examination data in the mean QLQ-C30 functional scale scores, and the use of the molecular targeted therapy after WBRT in the mean QLQ-BN20 scores (Table 4).

\section{Discussion}

The avoidance of WBRT is a current trend in some clinical situations [15], but WBRT is still important for most cases of brain metastases [16]. Recent prospective studies demonstrated that the use of WBRT is more frequently associated with cognitive side effects compared to stereotactic radiosurgery (SRS) alone. However, the role of WBRT is to improve the intracranial tumor control, and there is possibility that NCF deteriorate due to progression intracranial tumor by omitting WBRT [2]. In the JROSG 99-1 Study by Aoyama et al., the patients assigned to WBRT+SRS group demonstrated better short-term NCF compared with those assigned to SRS alone group. On the other hand, in this study, intracranial tumor control has been shown to be related to NCF deterioration at 4 months. Moreover, the reported percentage 


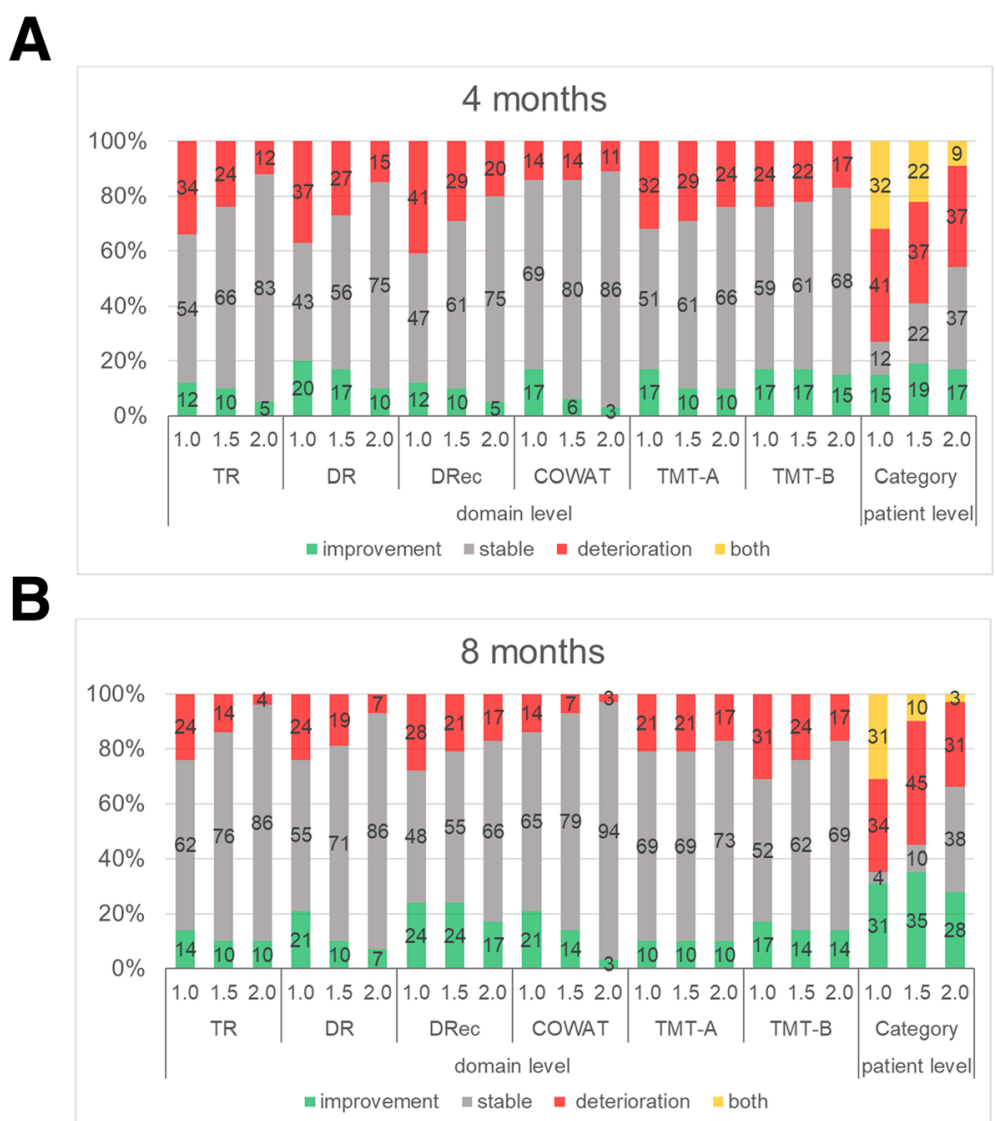

Fig. 1 Changes in the patients' NCF scores from baseline to 4 months (a) and from baseline to 8 months (b) at the domain level and at the patient level with the use of three cut-off values. TR: Total Recall domain of the Hopkins Verbal Learning Test-Revised (HVLT-R), DR: Delayed Recall domain of the HVLT-R, DRec: the Delayed Recognition domain of the HVLT-R, COWAT: the Control Oral Word Association Test, TMT-A: part A of the Trail-Making Test (TMT), TMT-B: part B of the TMT

of patients suffering neurocognitive deterioration varies widely among the publications because of the lack of standardization in the NCF score change criteria. In the Alliance trial comparing WBRT+SRS and SRS-alone, cognitive deterioration was defined as deterioration over 1.0 SD from baseline on at least one domain at 3 months post-WBRT. That trial's authors Brown PD et al. reported that cognitive deterioration was seen in 91.7\% of the patients in the WBRT+SRS arm and $63.5 \%$ in the SRS-alone arm $(p<0.001)$ [6]. In the MD Anderson Cancer Center trial in the same setting, $52 \%$ of the patients in the WBRT+SRS arm and $24 \%$ in the SRS-alone arm showed neurocognitive deterioration at 24 weeks after treatment [7]. In that trial, a 5-point drop in any domain of the HVLT-R was defined as deterioration. In the RTOG 0614 study comparing WBRT with or without memantine, the cut off value for cognitive failure were 2SD and the reliable change index [17]. The probability of cognitive function failure at 24 weeks was $53.8 \%$ in the memantine arm and $64.9 \%$ in the placebo arm $(p=0.01)$ [8].
There are the two pitfalls in interpreting these data. One is that some patients in the deterioration group could have both deterioration in one or more domains and improvement in other domains. These 'both' patients could be included in an improvement group if the 'improvement on at least one domain' definition was applied. In a study that assessed the changes in NCF after SRS alone, van der Meer et al. used the cut-off of $1.5 \mathrm{SD}$ and categorized their patients into four groups: improvement (14\%), stable (67\%), deterioration (14\%), and both (6\%) at 3 months after the initial SRS [11]. In the present study, as much as $32 \%$ of the patients (with 1.0 SD as the cut-off), 22\% (with $1.5 \mathrm{SD}$ ), or $9 \%$ (with $2.0 \mathrm{SD}$ ) showed both deterioration and improvement at 4 months. When these 'both' patients are included in the deterioration group or the improvement group with the cut-off of $1.0 \mathrm{SD}$, the percentage of patients with deterioration and the percentage of those with improvement increase from 41 to $73 \%(41 \%+32 \%)$ and from 15 to $47 \%(15 \%+32 \%)$, respectively. 


\section{A}

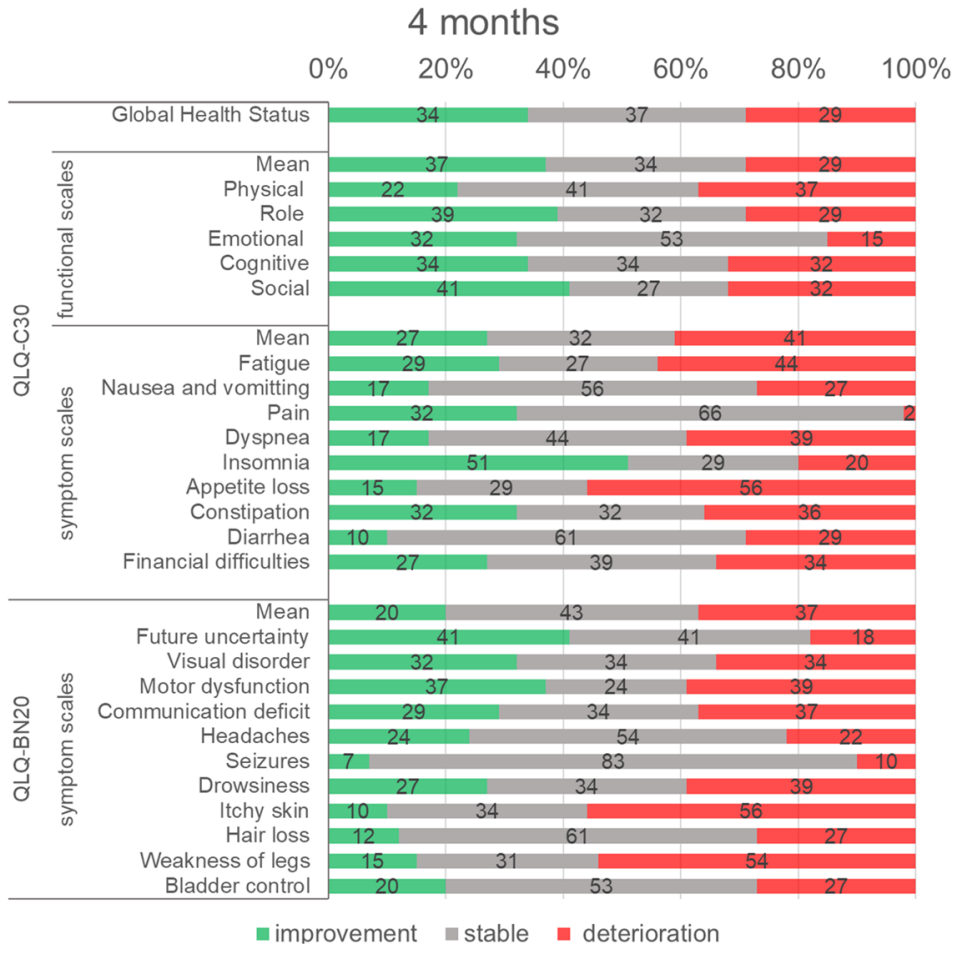

B

\section{8 months}

$0 \%$

$20 \% \quad 40 \%$

$60 \%$

$80 \%$

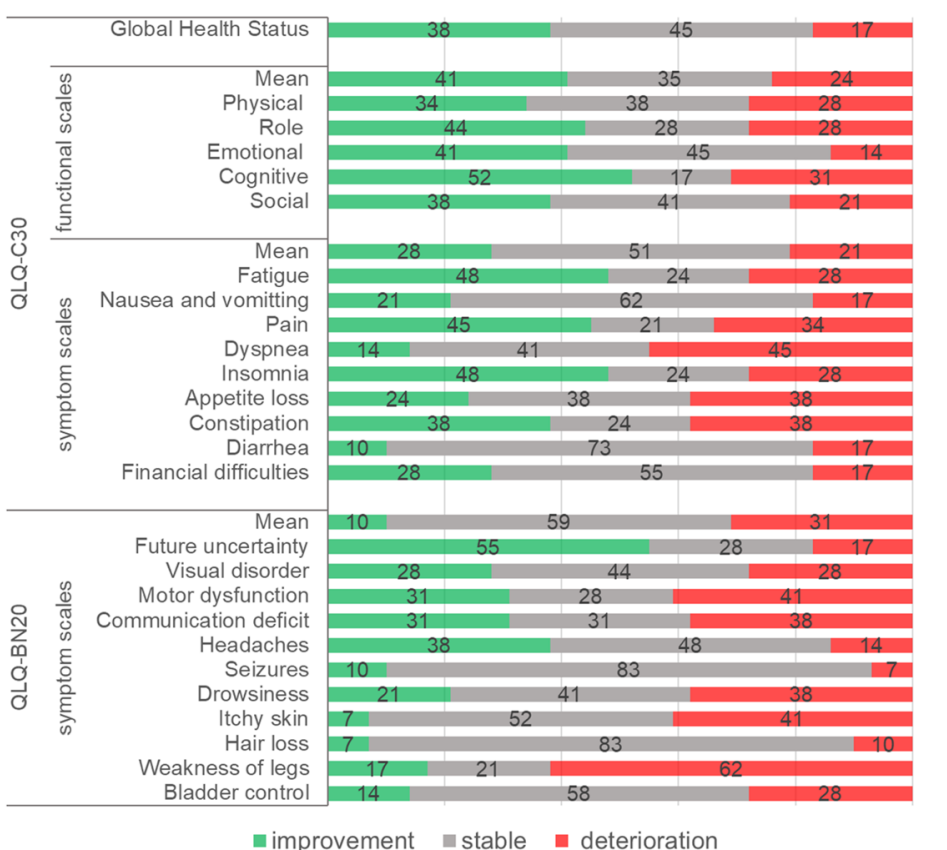

Fig. 2 Changes in the patients'HR-QOL scores from baseline to 4 months (a) and from baseline to 8 months (b). QLQ-C30: European Organization for Research and Treatment of Cancer (EORTC) Quality of Life Questionnaire Core 30. QLQ-BN20: European Organization for Research and Treatment of Cancer (EORTC) Brain Cancer Module 
Table 3 Number of patients with $\geq 10$-point score deterioration and improvement in HR-QOL from baseline in each category of NCF by using three cut-off values at 4 months

\begin{tabular}{|c|c|c|c|c|c|c|}
\hline \multirow[b]{2}{*}{ DETERIORATION } & \multicolumn{4}{|c|}{ n (\%: n/No. of patient category) } & \multirow{2}{*}{$\begin{array}{l}p \text {-value } \\
\text { Deterioration vs. others }\end{array}$} & \multirow{2}{*}{$\begin{array}{l}p \text {-value } \\
\text { Deterioration/Both vs. others }\end{array}$} \\
\hline & Improvement & Stable & Deterioration & Both & & \\
\hline \multicolumn{7}{|l|}{ QLQ-C30: } \\
\hline \multicolumn{7}{|c|}{ Global Health Status } \\
\hline $1.0 \mathrm{SD}$ & $2(33)$ & $2(40)$ & $4(24)$ & $4(31)$ & 0.729 & 0.701 \\
\hline $1.5 \mathrm{SD}$ & $2(25)$ & $4(44)$ & $3(20)$ & $3(33)$ & 0.480 & 0.507 \\
\hline $2.0 \mathrm{SD}$ & $1(14)$ & $6(40)$ & $3(20)$ & $2(50)$ & 0.480 & 0.744 \\
\hline \multicolumn{7}{|c|}{ Functional scales } \\
\hline $1.0 \mathrm{SD}$ & $1(17)$ & $1(20)$ & $9(53)$ & $1(8)$ & $0.013^{*}$ & 0.457 \\
\hline $1.5 \mathrm{SD}$ & $1(13)$ & $2(22)$ & $8(53)$ & $1(11)$ & $0.015^{*}$ & 0.296 \\
\hline $2.0 \mathrm{SD}$ & $1(14)$ & $3(20)$ & $7(47)$ & $1(25)$ & 0.083 & 0.168 \\
\hline \multicolumn{7}{|c|}{ Symptom scales } \\
\hline $1.0 \mathrm{SD}$ & $2(33)$ & $1(20)$ & $8(47)$ & $4(31)$ & 0.328 & 0.716 \\
\hline $1.5 \mathrm{SD}$ & $2(25)$ & $3(33)$ & $7(47)$ & $3(33)$ & 0.336 & 0.519 \\
\hline $2.0 \mathrm{SD}$ & $2(29)$ & $6(40)$ & $6(40)$ & $1(25)$ & 0.749 & 1.000 \\
\hline \multicolumn{7}{|l|}{ QLQ-BN20: } \\
\hline $1.0 \mathrm{SD}$ & $2(33)$ & $0(0)$ & $8(47)$ & $5(39)$ & 0.328 & 0.168 \\
\hline $1.5 \mathrm{SD}$ & $2(25)$ & $2(22)$ & $7(47)$ & $4(44)$ & 0.336 & 0.195 \\
\hline $2.0 \mathrm{SD}$ & $2(29)$ & $4(27)$ & $7(47)$ & $2(50)$ & 0.336 & 0.211 \\
\hline IMPROVEMENT & Improvement & Stable & Deterioration & Both & Improvement vs. others & Improvement/Both vs. others \\
\hline \multicolumn{7}{|l|}{ QLQ-C30: } \\
\hline \multicolumn{7}{|c|}{ Global Health Status } \\
\hline $1.0 \mathrm{SD}$ & $3(50)$ & $1(20)$ & $1(20)$ & $5(39)$ & 0.393 & 0.346 \\
\hline $1.5 \mathrm{SD}$ & $3(38)$ & $2(22)$ & $2(22)$ & $5(56)$ & 1.000 & 0.189 \\
\hline $2.0 \mathrm{SD}$ & $3(43)$ & $4(27)$ & $4(27)$ & $2(50)$ & 0.673 & 0.463 \\
\hline \multicolumn{7}{|c|}{ Functional scales } \\
\hline $1.0 \mathrm{SD}$ & $4(67)$ & $1(20)$ & $1(20)$ & $4(31)$ & 0.168 & 0.533 \\
\hline $1.5 \mathrm{SD}$ & $5(63)$ & $3(33)$ & $3(33)$ & $2(22)$ & 0.117 & 0.745 \\
\hline $2.0 \mathrm{SD}$ & $4(57)$ & $5(33)$ & $5(33)$ & $1(25)$ & 0.390 & 0.491 \\
\hline \multicolumn{7}{|c|}{ Symptom scales } \\
\hline $1.0 \mathrm{SD}$ & $2(33)$ & $1(20)$ & $1(20)$ & $3(23)$ & 0.651 & 1.000 \\
\hline $1.5 \mathrm{SD}$ & $3(38)$ & $3(33)$ & $3(33)$ & $2(22)$ & 0.658 & 1.000 \\
\hline $2.0 \mathrm{SD}$ & $2(29)$ & $4(27)$ & $4(27)$ & $1(25)$ & 1.000 & 1.000 \\
\hline \multicolumn{7}{|l|}{ QLQ-BN20: } \\
\hline $1.0 \mathrm{SD}$ & $3(50)$ & $1(20)$ & $1(20)$ & $1(8)$ & 0.077 & 1.000 \\
\hline $1.5 \mathrm{SD}$ & $4(50)$ & $1(11)$ & $1(11)$ & $0(0)$ & $0.033^{*}$ & 0.698 \\
\hline $2.0 \mathrm{SD}$ & $2(29)$ & $3(20)$ & $3(20)$ & $0(0)$ & 0.606 & 1.000 \\
\hline
\end{tabular}

${ }^{*} p$-value $<0.05$

Both significant improvement and deterioration were observed in different NCF domains HR-QOL health-related quality of life, NCF neurocognitive function. QLQC30 European Organization for Research and Treatment of Cancer (EORTC) Quality of Life Questionnaire Core 30. QLQ-BN20 European Organization for Research and Treatment of Cancer (EORTC) Brain Cancer Module

The other pitfall in the data interpretation is that the cutoff value is different for each study. Therefore, we tried to construct the NCF change criteria based on HR-QOL because the goal of the treatment of brain metastases is the maintenance or even improvement of the patient's HR-QOL, considering the palliative nature of the treatment. In addition, it is known that NCF and HR-QOL are related after WBRT for brain metastasis [18]. It should be noted that assessments of 'clinically meaningful changes in HR-QOL status' is another topic for research, but we used the HR- 
Table 4 Percentage of patients divided into two groups (age, GPA, number of examinations, intracranial control at 4 months, Systematic therapies prior WBRT, Cytotoxic chemotherapy after WBRT, and Molecular targeted therapy after WBRT) with deterioration in NCF scores with 1.5 SD and HR-QOL scores at 4 months

\begin{tabular}{|c|c|c|c|c|c|c|c|c|c|c|c|c|}
\hline \multirow[t]{2}{*}{ Factors } & \multicolumn{2}{|c|}{ Age group, \% } & \multirow[t]{2}{*}{$p$} & \multicolumn{2}{|l|}{ GPA, \% } & \multirow[t]{2}{*}{$p$} & \multicolumn{2}{|c|}{ No. of examinations, \% } & \multirow[t]{2}{*}{$p$} & \multicolumn{2}{|c|}{ Intracranial control at 4 mos., $\%$} & \multirow[t]{2}{*}{$p$} \\
\hline & $<65$ & $\geq 65$ & & $4.0-2.0$ & $1.5-0.0$ & & 3 & 2 & & Non-PD & PD & \\
\hline \multicolumn{13}{|l|}{ NCF: } \\
\hline TR & 33 & 15 & 0.28 & 24 & 25 & 1.00 & 14 & 50 & $0.04^{*}$ & 8 & 62 & $<0.01^{*}$ \\
\hline DR & 38 & 15 & 0.16 & 18 & 33 & 0.31 & 14 & 58 & $<0.01^{*}$ & 15 & 46 & 0.06 \\
\hline DRec & 29 & 30 & 1.00 & 29 & 29 & 1.00 & 17 & 58 & $0.02^{*}$ & 23 & 39 & 0.45 \\
\hline COWAT & 14 & 10 & 1.00 & 24 & 4 & 0.14 & 10 & 17 & 0.62 & 8 & 23 & 0.31 \\
\hline TMT-A & 24 & 35 & 0.51 & 29 & 29 & 1.00 & 21 & 50 & 0.13 & 23 & 46 & 0.16 \\
\hline TMT-B & 19 & 25 & 0.72 & 18 & 25 & 0.71 & 10 & 50 & $0.01^{*}$ & 4 & 54 & $<0.01^{*}$ \\
\hline \multicolumn{13}{|l|}{ HR-QOL: } \\
\hline \multicolumn{13}{|l|}{ QLQ-C30; } \\
\hline Global Health Status & 29 & 30 & 1.00 & 29 & 29 & 1.00 & 31 & 25 & 1.00 & 35 & 15 & 0.28 \\
\hline Functional scales & 29 & 30 & 1.00 & 18 & 38 & 0.30 & 17 & 58 & $0.02^{*}$ & 19 & 46 & 0.13 \\
\hline Symptom scales & 43 & 30 & 0.52 & 41 & 33 & 0.75 & 35 & 42 & 0.73 & 35 & 39 & 1.00 \\
\hline QLQ-BN20 & 33 & 40 & 0.75 & 41 & 33 & 0.75 & 35 & 42 & 0.73 & 35 & 31 & 1.00 \\
\hline \multirow[t]{2}{*}{ Factors } & \multicolumn{3}{|c|}{$\begin{array}{l}\text { Systemic therapies } \\
\text { prior to WBRT, \% }\end{array}$} & $p$ & \multicolumn{3}{|c|}{$\begin{array}{l}\text { Cytotoxic chemotherapy } \\
\text { after WBRT, } \%\end{array}$} & $p$ & \multicolumn{3}{|c|}{ Molecular targeted therapy after WBRT, \% } & \multirow[t]{2}{*}{$p$} \\
\hline & No & Yes & & & No & Yes & & & No & Yes & & \\
\hline \multicolumn{13}{|l|}{ NCF: } \\
\hline $\mathrm{TR}$ & 13 & 32 & & 0.27 & 27 & 21 & & 0.73 & 18 & 32 & & 0.47 \\
\hline DR & 19 & 32 & & 0.48 & 27 & 26 & & 1.00 & 23 & 32 & & 0.73 \\
\hline DRec & 25 & 32 & & 0.73 & 23 & 37 & & 0.49 & 27 & 32 & & 1.00 \\
\hline COWAT & 13 & 12 & & 1.00 & 14 & 11 & & 1.00 & 18 & 5 & & 0.35 \\
\hline TMT-A & 31 & 28 & & 1.00 & 18 & 42 & & 0.17 & 36 & 21 & & 0.33 \\
\hline TMT-B & 6 & 32 & & 0.07 & 23 & 21 & & 1.00 & 18 & 26 & & 0.71 \\
\hline \multicolumn{13}{|l|}{ HR-QOL: } \\
\hline \multicolumn{13}{|l|}{ QLQ-C30; } \\
\hline Global Health Status & 31 & 28 & & 1.00 & 36 & 21 & & 0.33 & 36 & 21 & & 0.33 \\
\hline Functional scales & 19 & 36 & & 0.31 & 23 & 37 & & 0.49 & 32 & 26 & & 0.74 \\
\hline Symptom scales & 38 & 36 & & 1.00 & 36 & 37 & & 1.00 & 46 & 26 & & 0.33 \\
\hline QLQ-BN20 & 38 & 36 & & 1.00 & 32 & 42 & & 0.53 & 55 & 16 & & $0.02^{*}$ \\
\hline
\end{tabular}

${ }^{*} p$-value $<0.05$ NCF neurocognitive function, TR Totall Recall domain of the Hopkins Verbal Learning Test-Revised (HVLT-R), DR Delayed Recall domain of the HVLT-R, DRec the Delayed Recognition domain of the HVLT-R, COWAT the Control Oral Word Association Test, TMT-A part A of the Trail-Making Test (TMT), TMT-B part B of the TMT, HR-QOL health-related quality of life, GPA Graded Prognosis Assessment, PD progressive disease. QLQ-C30 European Organization for Research and Treatment of Cancer (EORTC) Quality of Life Questionnaire Core 30. QLQ-BN20 European Organization for Research and Treatment of Cancer (EORTC) Brain Cancer Module

QOL cut-off value of $\geq 10$ points, which reflects a moderate change in the HR-QOL status in the QLQ-C30 and the QLQ-BN20 [19, 20]. In the present analyses, based on the patients' mean QLQ-C30 functional scale scores with 1.0 SD and 1.5SD, a clinically meaningful deterioration of the patients' HR-QOL was observed significantly more frequently in the NCF deterioration group compared to the NCF improvement group, stable group, and both-deterioration-and-improvement groups at 4 months.

On the other hand, a clinically meaningful improvement of the patients' HR-QOL was observed significantly more frequently in the NCF improvement group compared to the other three patient groups based on the mean of the QLQ-BN20 scores with $1.5 \mathrm{SD}$ at 4 months. However, when the 'both' patients were included in the deterioration group or the improvement group, the statistical significance of all of the above findings was lost. This implies that patients who show both deterioration and improvement in different domains should be differentiated from patients who exhibit only deterioration or only improvement. Therefore, the use of 'at least one 
domain' for the definition of deterioration/improvement should be interpreted carefully, and the automatic inclusion of these 'both' patients in a deterioration group should be questioned.

Our present findings demonstrate that the use of a stricter cut-off value (i.e., 2.0 SD) resulted in a reduction of the number of 'both' patients but provided less sensitivity, and it could not detect significant HR-QOL changes. We thus propose that the cut-off value of $1.5 \mathrm{SD}$ and the exclusion of 'both' patients from the deterioration and improvement groups would most closely reflect the clinically meaningful changes in HR-QOL.

Our study has several limitations. First, although the examination compliance in our study population was better (85\% at 4 months) than that reported in other studies (59$73 \%$ at $2-4$ months) $[6,8,11,21-23]$, there were patients who could not perform the follow up examinations. Second, this study was unable to perform multivariate analysis that considers confounding factors related to cognitive dysfunction such as opioids [24], chemotherapy [25], surgery, etc.. However, in this study patients group, it has been confirmed that the presence or absence of surgery/chemotherapy did not cause a significant difference in NCF tests at baseline. Third, the prognoses of our study population were heterogeneous, since patients who have undergone WBRT postoperatively and patients with meningeal carcinomatosis were analyzed together. The prognosis of individuals with brain metastases has been prolonged, and it may be necessary to change the time points of the NCF and HR-QOL examinations according to patients' prognoses. Finally, due to the small sample size, we could not fully analyze the patients' NCF and HR-QOL after 8 months post-WBRT. It is necessary to verify our present findings with a larger number of patients over longer follow-up periods.

\section{Conclusions}

The use of $1.5 \mathrm{SD}$ as the cut-off for NCF best reflected the patients' HR-QOL status. The inclusion of 'both' in either a 'deterioration' or 'improvement' group blurs the changes in NCF reflecting the clinical meaningful changes in HRQOL, and therefore patients showing both deterioration and improvement should not be included in either group.

\section{Supplementary information}

Supplementary information accompanies this paper at https://doi.org/10. 1186/s12885-020-6559-3.

Additional file 1: Figure S1. Overall survival in the patients who underwent examinations at baseline only, at baseline and 4 months only, or at baseline, 4 months and 8 months. BL: baseline. MST: median survival time. WBRT: whole-brain radiotherapy.

Additional file 2: Table S1. The mean scores in examinations at baseline and comparisons of pairs of groups. Table S2. Including full scales/items, Number of patients with $\geq 10$ score deterioration and improvement in HR-QOL from BL in each category of NCF by using three cut-off values at 4 months.

\section{Abbreviations}

COWAT: Control Oral Word Association Test; DR: Delayed Recall;

DRec: Delayed Recognition; EORTC: European Organization for Research and Treatment of Cancer; GHS: global health status; GPA: Graded Prognostic Assessment; HR-QOL: Health-related quality of life; HVLT-R: Hopkins Verbal Learning Test-Revised; KPS: Karnofsky Performance Status; MST: median survival time; NCF: Neurocognitive function; PD: progressive disease; QLQBN20: Quality of Life Questionnaire Brain Cancer Module; QLQ-C30: Quality of Life Questionnaire Core 30; RANO: Response Assessment in Neuro-Oncology; SDs: standard deviations; SRS: stereotactic radiosurgery; TMT: Trail-Making Test; TMT-A: Parts A of the TMT; TMT-B: Parts B of the TMT; TR: Total Recall; WBRT: whole-brain radiation therapy

\section{Acknowledgements}

Conducting each NCF tests and HR-QOL questionnaires: Yukiko Morita, our research assistant, Department of Radiology and Radiation Oncology, Niigata University Graduate School of Medical and Dental Sciences, 1-757

Asahimachi-dori, Chuo-ku, Niigata 951-8510, Japan.

\section{Authors' contributions}

Conception and design: TN, HA, HS. Provision of study materials: TN, HA, HS, KT, KM, TO, AO, EA, MK. Collection and assembly of data: TN. Data analysis and. Interpretation: TN, HA, HS. Manuscript writing: TN, HA, HS, ST. Final approval of manuscript: All authors.

\section{Funding}

This work was partially supported by Japan Society for the Promotion of Science (JSPS) KAKENHI Grant No. $15 \mathrm{H} 04903$.

\section{Availability of data and materials}

The datasets generated during and/or analysed during the current study are available from the corresponding author on reasonable request.

\section{Ethics approval and consent to participate}

This study was approved by the Institutional Review Board of Niigata University Hospital (Study \#1449). Written informed consent was obtained from all patients.

\section{Consent for publication}

Not applicable.

\section{Competing interests}

The authors declare that they have no competing interests.

\section{Author details}

${ }^{1}$ Department of Radiology and Radiation Oncology, Niigata University Graduate School of Medical and Dental Sciences, 1-757 Asahimachi-dori, Chuo-ku, Niigata 951-8510, Japan. ²Department of Radiation Oncology, Niigata University Medical and Dental hospital, Niigata, Japan.

Received: 7 July 2019 Accepted: 20 January 2020

Published online: 29 January 2020

References

1. Barnholtz-Sloan JS, Yu C, Sloan AE, Vengoechea J, Wang M, Dignam JJ, Vogelbaum MA, Sperduto PW, Mehta MP, Machtay M, et al. A nomogram for individualized estimation of survival among patients with brain metastasis. Neuro-oncology. 2012;14(7):910-8.

2. Aoyama H, Tago M, Kato N, Toyoda T, Kenjyo M, Hirota S, Shioura H, Inomata T, Kunieda E, Hayakawa K, et al. Neurocognitive function of patients with brain metastasis who received either whole brain radiotherapy plus stereotactic radiosurgery or radiosurgery alone. Int J Radiat Oncol Biol Phys. 2007;68(5):1388-95.

3. Li J, Bentzen SM, Renschler M, Mehta MP. Regression after whole-brain radiation therapy for brain metastases correlates with survival and improved neurocognitive function. J Clin Oncol. 2007;25(10):1260-6. 
4. Lin NU, Wefel JS, Lee EQ, Schiff D, van den Bent MJ, Soffietti R, Suh JH, Vogelbaum MA, Mehta MP, Dancey J, et al. Challenges relating to solid tumour brain metastases in clinical trials, part 2: neurocognitive, neurological, and quality-of-life outcomes. A report from the RANO group. Lancet Oncol. 2013;14(10):e407-16.

5. Schimmel WCM, Gehring K, Eekers DBP, Hanssens PEJ, Sitskoorn MM. Cognitive effects of stereotactic radiosurgery in adult patients with brain metastases: a systematic review. Adv Radiat Oncol. 2018;3(4):568-81.

6. Brown PD, Jaeckle K, Ballman KV, Farace E, Cerhan JH, Anderson SK, Carrero XW, Barker FG 2nd, Deming R, Burri SH, et al. Effect of radiosurgery alone vs radiosurgery with whole brain radiation therapy on cognitive function in patients with 1 to 3 brain metastases: a randomized clinical trial. Jama. 2016; 316(4):401-9.

7. Chang EL, Wefel JS, Hess KR, Allen PK, Lang FF, Kornguth DG, Arbuckle RB, Swint JM, Shiu AS, Maor MH, et al. Neurocognition in patients with brain metastases treated with radiosurgery or radiosurgery plus whole-brain irradiation: a randomised controlled trial. Lancet Oncol. 2009;10(11):1037-44.

8. Brown PD, Pugh S, Laack NN, Wefel JS, Khuntia D, Meyers C, Choucair A, Fox S, Suh JH, Roberge D, et al. Memantine for the prevention of cognitive dysfunction in patients receiving whole-brain radiotherapy: a randomized, double-blind, placebo-controlled trial. Neuro-oncology. 2013;15(10):1429-37.

9. Mitrushina M, Boone KB, Razani J, D'Elia LF. Handbook of Normative Data for Neuropsychological Assessment, Second Edition. USA: Oxford University Press; 2005

10. Ito $E$, Hatta $T$, Ito $Y$, Kogure $T$, Watanabe H. Performance of verbal fluency tasks in Japanese healthy adults -effect of gender, age and education on the performance. Jpn J Neuropsychol. 2004;20(4):254-63.

11. van der Meer PB, Habets EJJ, Wiggenraad RG, Verbeek-de Kanter A, Lycklama ANGJ, Zwinkels H, Klein M, Dirven L, Taphoorn MJB. Individual changes in neurocognitive functioning and health-related quality of life in patients with brain oligometastases treated with stereotactic radiotherapy. J Neuro-Oncol. 2018;139(2):359-68.

12. Aaronson NK, Ahmedzai S, Bergman B, Bullinger M, Cull A, Duez NJ, Filiberti A, Flechtner $\mathrm{H}$, Fleishman SB, de Haes JC, et al. The European Organization for Research and Treatment of Cancer QLQ-C30: a quality-of-life instrument for use in international clinical trials in oncology. J Natl Cancer Inst. 1993; 85(5):365-76.

13. Taphoorn MJ, Claassens L, Aaronson NK, Coens C, Mauer M, Osoba D, Stupp R, Mirimanoff RO, van den Bent MJ, Bottomley A. An international validation study of the EORTC brain cancer module (EORTC QLQ-BN20) for assessing health-related quality of life and symptoms in brain cancer patients. Eur J Cancer. 2010;46(6):1033-40.

14. Sperduto PW, Kased N, Roberge D, Xu Z, Shanley R, Luo X, Sneed PK, Chao ST, Weil RJ, Suh J, et al. Summary report on the graded prognostic assessment: an accurate and facile diagnosis-specific tool to estimate survival for patients with brain metastases. J Clin Oncol. 2012;30(4):419-25.

15. Brown PD, Ahluwalia MS, Khan OH, Asher AL, Wefel JS, Gondi V. Wholebrain radiotherapy for brain metastases: evolution or revolution? J Clin Oncol. 2018;36(5):483-91.

16. Mehta MP, Aoyama H, Gondi V. The changing role of whole-brain radiotherapy: demise or time for selective usage? JAMA Oncol. 2017; 3(8):1021-2.

17. Jacobson NS, Truax P. Clinical significance: a statistical approach to defining meaningful change in psychotherapy research. J Consult Clin Psychol. 1991; 59(1):12-9.

18. Li J, Bentzen SM, Li J, Renschler M, Mehta MP. Relationship between neurocognitive function and quality of life after whole-brain radiotherapy in patients with brain metastasis. Int J Radiat Oncol Biol Phys. 2008;71(1):64-70.

19. Maringwa J, Quinten C, King M, Ringash J, Osoba D, Coens C, Martinelli F, Reeve BB, Gotay C, Greimel E, et al. Minimal clinically meaningful differences for the EORTC QLQ-C30 and EORTC QLQ-BN20 scales in brain cancer patients. Ann Oncol. 2011;22(9):2107-12.

20. Osoba D, Rodrigues G, Myles J, Zee B, Pater J. Interpreting the significance of changes in health-related quality-of-life scores. J Clin Oncol. 1998;16(1):139-44.

21. Steinmann D, Paelecke-Habermann Y, Geinitz H, Aschoff R, Bayerl A, Bolling T, Bosch E, Bruns F, Eichenseder-Seiss U, Gerstein J, et al. Prospective evaluation of quality of life effects in patients undergoing palliative radiotherapy for brain metastases. BMC Cancer. 2012;12:283.

22. Soffietti $R$, Kocher M, Abacioglu UM, Villa S, Fauchon F, Baumert BG, Fariselli L, Tzuk-Shina T, Kortmann RD, Carrie C, et al. A European Organisation for
Research and Treatment of Cancer phase III trial of adjuvant whole-brain radiotherapy versus observation in patients with one to three brain metastases from solid tumors after surgical resection or radiosurgery: quality-of-life results. J Clin Oncol. 2013;31(1):65-72.

23. Kepka L, Tyc-Szczepaniak D, Osowiecka K, Sprawka A, Trabska-Kluch B, Czeremszynska B. Quality of life after whole brain radiotherapy compared with radiosurgery of the tumor bed: results from a randomized trial. Clin Transl Oncol. 2018;20(2):150-9.

24. Lawlor PG. The panorama of opioid-related cognitive dysfunction in patients with cancer: a critical literature appraisal. Cancer. 2002;94(6): 1836-53.

25. Lange M, Rigal O, Clarisse B, Giffard B, Sevin E, Barillet M, Eustache F, Joly F. Cognitive dysfunctions in elderly cancer patients: a new challenge for oncologists. Cancer Treat Rev. 2014;40(6):810-7.

\section{Publisher's Note}

Springer Nature remains neutral with regard to jurisdictional claims in published maps and institutional affiliations.
Ready to submit your research? Choose BMC and benefit from:

- fast, convenient online submission

- thorough peer review by experienced researchers in your field

- rapid publication on acceptance

- support for research data, including large and complex data types

- gold Open Access which fosters wider collaboration and increased citations

- maximum visibility for your research: over $100 \mathrm{M}$ website views per year

At BMC, research is always in progress.

Learn more biomedcentral.com/submissions 